\title{
AN ADAPTIVE/PREDICTIVE APPROACH FOR RUNOFF TEMPORAL BEHAVIOUR OF HIGH DEPENDENCE RIVERS
}

\author{
Molina, José-Luis ${ }^{(1)\left({ }^{*}\right)}$, Zazo, Santiago ${ }^{(2)}$, Martín, Ana-María (3)
}

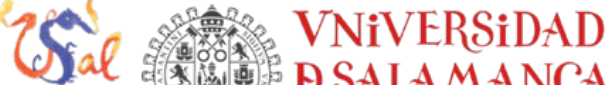 isaLAMANCA}

Methodology applied

Stage $-1^{\text {st. }}$ Statistical Analysis of the historical series of run-off and ARMA $(1,1)$ Model Definition \begin{tabular}{c} 
Traditional Analysis \\
(Main statistical parameters and Correlogram) \\
\hline
\end{tabular} Bayesian Network Training

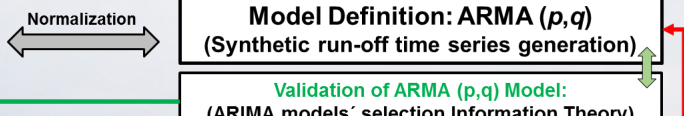
(ARIMA models' selection Information Theory)

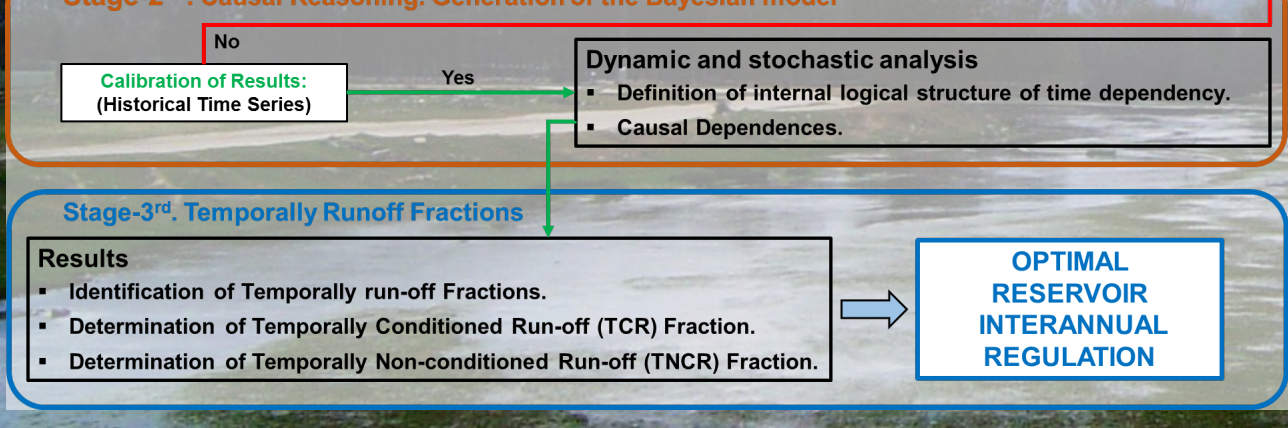

Stage-3rd. Temporally Runoff Fractions
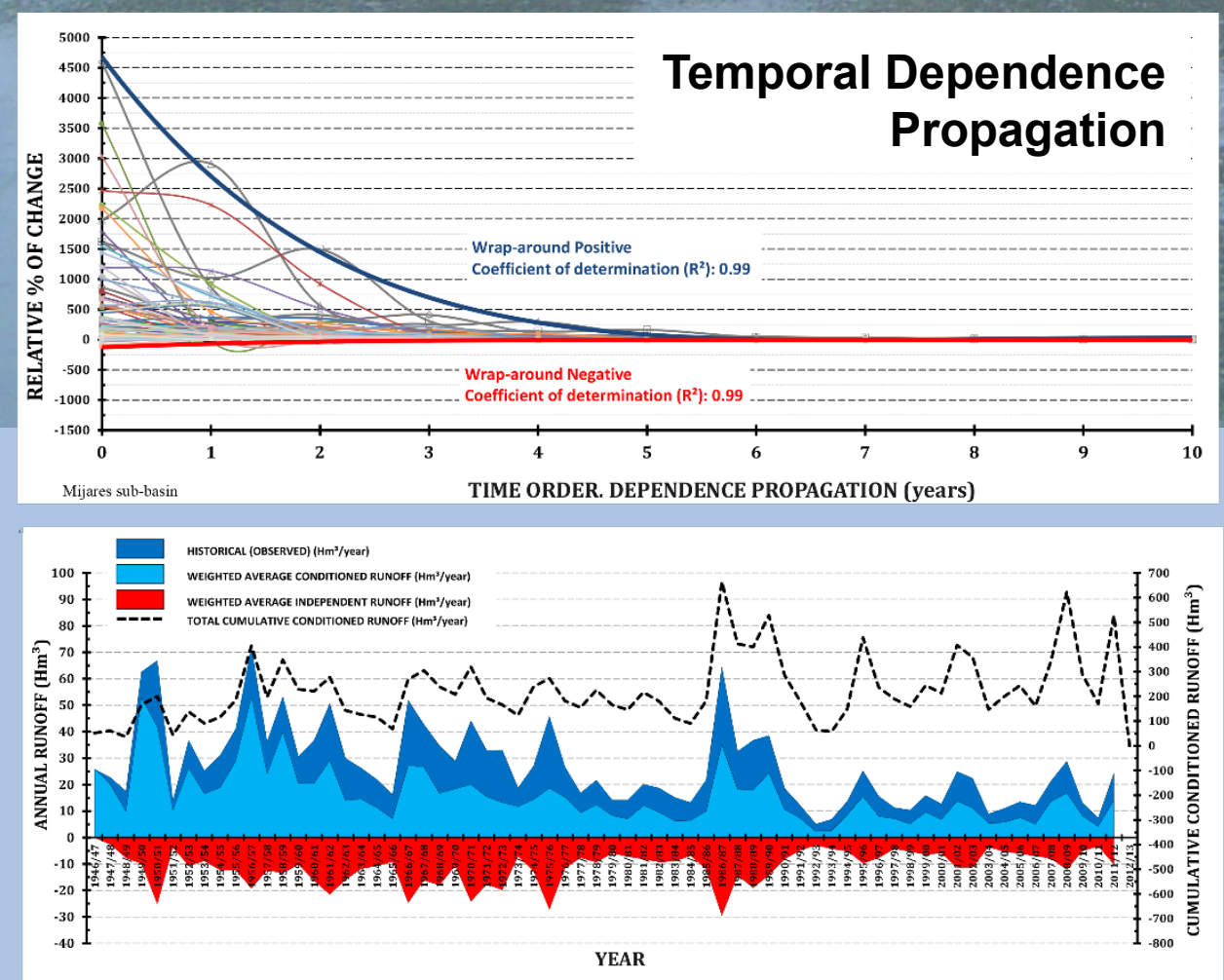

Temporally Runoff Fractions

\section{Stage -1 st. Statistical Analysis}
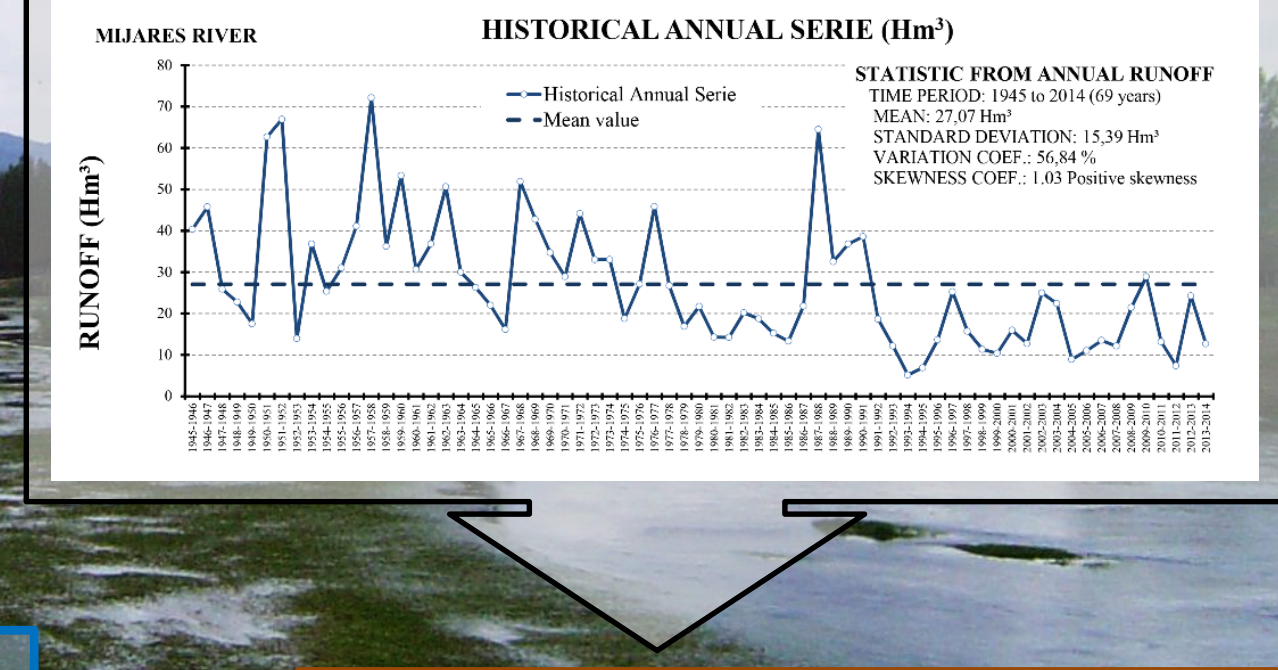

Stage-2 ${ }^{\text {nd }}$. Causal Reasoning

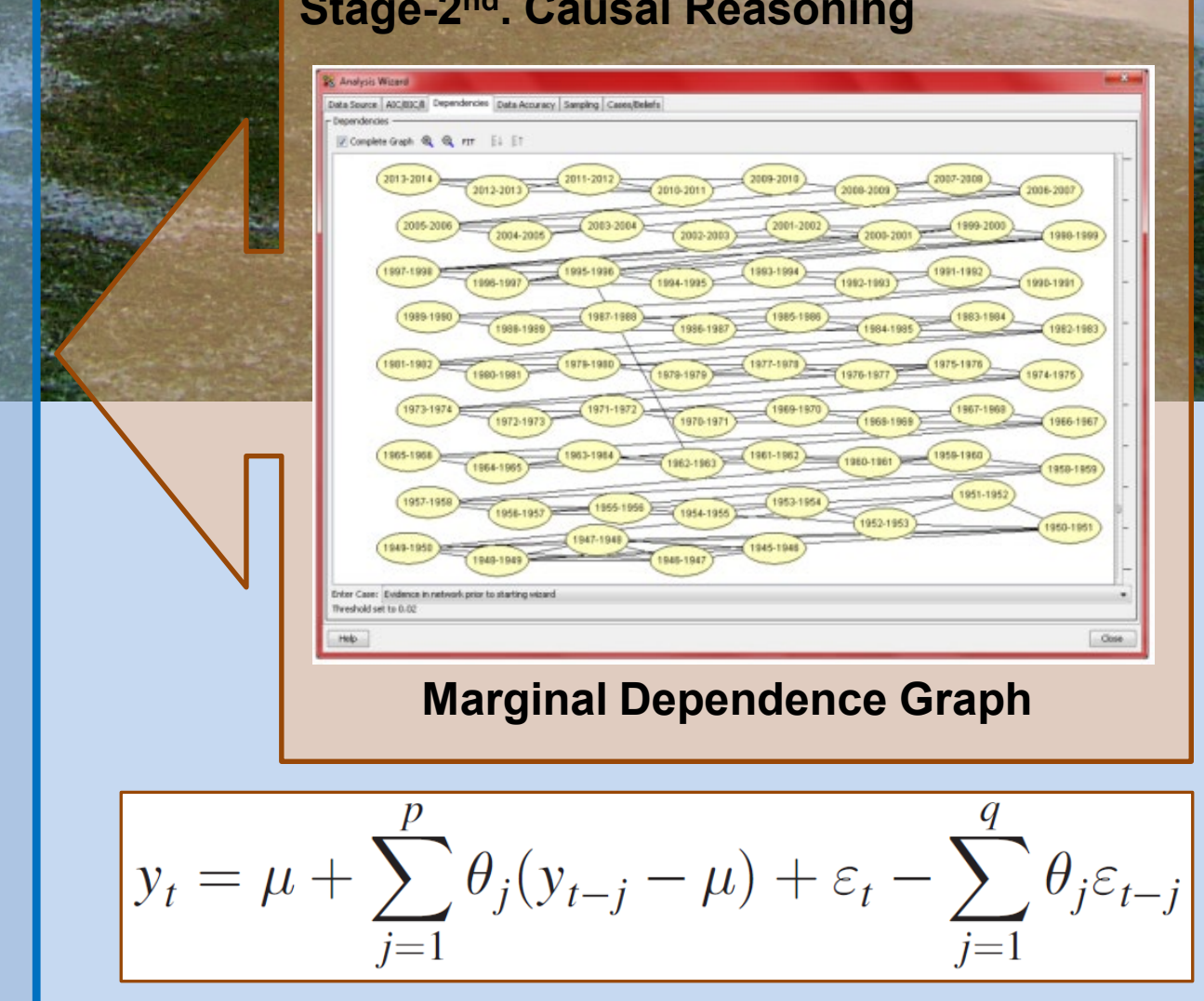

$\operatorname{ARMA}(p, q)$ model

\section{ABSTRACT}

A significant spatio-temporal alteration of traditional patterns for hydrological components' behavior is currently being observed. In this sense, a higher variability, more frequent and unpredictable extreme events (rainfall, flood and drought) occur in many areas. This is primarily due to global warming, which is producing a growing variability and uncertainty of water systems and specially, rivers' runoff. The understanding of these modifications and, consequently, this adaptive rivers' behavior is not trivial and requires new approaches incorporating dynamic and stochastic approaches. Causal Reasoning, supported by Bayesian modelling is a powerful stochastic approach to extract the time dependent logical structure that inherently underlies hydrological series. The river basin memory is dynamically and stochastically characterized in terms of the runoff dependence strength over the time. In this study, by means of causality, the Temporally Conditioned/Non-conditioned runoff (TCR/TNCR) fractions are identified and quantified. This research has important implications and applications, such as to the knowledge of the historical adaptive rivers' behavior, or to reservoirs' dimensioning optimization. This approach has been successfully applied to an unregulated river basin in Spain with a very high dependence temporal runoff behavior, within Júcar river basin (Mijares). Having a tool that could dynamically adjust the reservoir and/or channel capacity may help for reaching the optimal design and dimensioning of hydraulic infrastructures, which involves a lot of economic savings. Further work will largely comprise the introduction of the spatial dimension so the tool can integrated a full spatio-temporal analysis. The analyzed runoff behavior trends will be further used for building predictive models 